\title{
Tracking Analysis of Affine Projection Filters to Carrier Frequency Offset
}

\author{
Yong-Gang Zhu, Sheng-Yong Guan \\ Nanjing Telecommunication Technology Institute \\ Nanjing, China \\ zhumaka1982@163.com
}

\begin{abstract}
Carrier frequency offset (CFO) between transmitter and receiver carrier generators degrade the performance of adaptive filters seriously, even for very small CFO. Tracking analysis of the affine projection filter is carried out in the presence of CFO. The energy conservation relation of the affine projection filter with both random and cyclic nonstationarities is derived. Based on the energy conservation relation the steadystate mean-square error is derived in the presence of cyclic nonstationarities. The theoretical results are very close to the Monte Carlo simulation curves. Besides, the simulations results show that the tracking performance improves according to the increase of the order of the affine projection filters.
\end{abstract}

Keywords-adaptive filter; affine projection; tracking; carrier frequency offset; mean-square-error

\section{INTRODUCTION}

Adaptive filters are often used in nonstationary environments, such as wideband wireless digital communication systems, where they are required to track time variations in an unknown system or channel [1][2]. The ability of adaptive filters to track such variations affects the performance of these systems seriously. The variations of the system or channel can be classified as random system nonstationarities and cyclic system nonstationarities [2]. For the last decades, the ability of adaptive filters to track random system nonstationarities has received considerable attention [3][4][5][6]. The cyclic system nonstationarities, which is generated by the mismatches between the transmitter and receiver carrier generators, degrade the performance of adaptive filters seriously, even for ever small carrier frequency offsets. The ability of adaptive filtering algorithms to track such cyclic system variations is not yet fully understood. A recent contribution of Yousef in this regard [4], who presents a framework for the tracking analysis of adaptive algorithms that handles simultaneously both cyclic and random system nonstationarities. But this framework is restricted to scalar adaptive algorithms, such as Least Mean-Square (LMS) algorithm, Normalized LMS (NLMS) algorithm. Moinuddian solved the same problem for NLMS algorithm almost at the same time, independently [5].

As a generalization of the NLMS algorithm, the affine projection algorithm (APA) is considered to be a valid method to accelerate the convergence rate of adaptive filter especially when the input signal is correlated. And this algorithm has been researched deeply in recent years [7][8][9][10][11]. But,

\author{
Lan Zhao \\ Science and Technology on Communication Information \\ Security Control Laboratory \\ Jiaxing, China \\ hibry008@yahoo.com.cn
}

to our best knowledge, the tracking performance of the APA to cyclic system nonstationarities has received little attention. In this paper, the energy conservation relation of the APA is derived, under the condition of random and cyclic system nonstationarities. Then, based on the energy conservation relation, the tracking performance of the APA to the carrier frequency offset (CFO) is analyzed. This result is compared with the tracking performance of NLMS in [4][5] and the performance of the APA without CFO in [7]. Simulation results show that the analysis and the derived performance expressions are close to the simulation curves.

\section{DATA MODELS}

The system identification problem with channel fading and $\mathrm{CFO}$ is depicted in Fig. 1. Let $d(k)$ denotes the received signal in the wireless communication system:

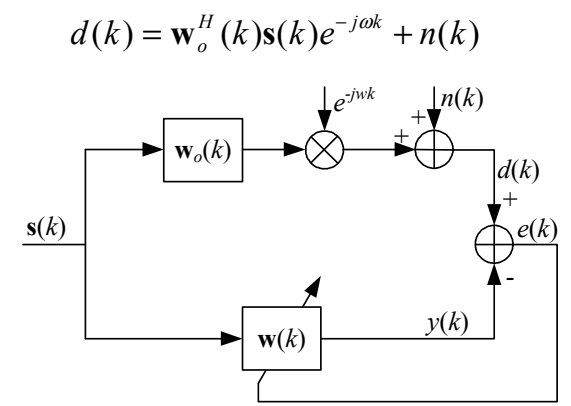

Figure1. System identification using an adaptive filter.

where $\mathbf{s}(k)$ is the transmitted signal vector with the power of $\sigma_{s}^{2}, \omega$ is carrier frequency offset, $n(k)$ is the channel noise with the variance of $\sigma_{n}^{2}$. Throughout this paper, the communication signal is assumed to be independent of both the noise and fading processes. And the noise is assumed to be additive white Gaussian noise (AWGN). $\mathbf{w}_{o}(k)$ is the unknown channel of finite impulse response of order $L$. It is assumed that the channel varies in time according to the rule

$$
\mathbf{w}_{o}(k)=\mathbf{w}_{o}+\boldsymbol{\theta}(k)
$$

where $\mathbf{w}_{o}$ is a constant vector, and $\boldsymbol{\theta}(k)$ is a random perturbation. In general, in the case of a fading channel, the perturbation $\boldsymbol{\theta}(k)$ can be modeled to a reasonable extent as an autoregressive (AR) process of order 1 [4]:

$$
\boldsymbol{\theta}(k+1)=\alpha \boldsymbol{\theta}(k)+\mathbf{q}(k)
$$

This work was supported by the Foundation of National Laboratory of Information Control Technology for Communication System of China. 
where $\alpha(0 \leq|\alpha|<1)$ is the regressive parameter. And $\mathbf{q}(n)$ is a zero-mean stationary random vector process with a positivedefinite covariance matrix $\mathbf{Q}=E\left\{\mathbf{q}(k) \mathbf{q}^{H}(k)\right\}$. $\mathbf{q}(n)$ satisfies the orthogonality theorem, when $\mathrm{m} \neq k, E\left\{\mathbf{q}(m) \mathbf{q}^{H}(k)\right\}=\mathbf{0}_{L \times L}$. If we set $\alpha=0$ and $\mathbf{q}(k)=\mathbf{0}_{L \times 1}$, then Eq. (1) is the model of the channel without random nonstationary. And if we set $\omega=0$, then Eq. (1) is the model of the channel without CFO. For a Rayleigh fading channel with maximum Doppler frequency $f_{D}$, one has $\alpha=I_{0}\left(2 \pi f_{D} T\right)$ and $\mathbf{Q}=\sqrt{1-\alpha^{2}} \mathbf{I}_{L \times L}$, where $I_{0}(\cdot)$ is the Bessel function of first kind and order zero, $T$ is the sampling period of the digital communication system and $\mathbf{I}$ is the identity matrix.

\section{ENERGY CONSERVATION RELATION OF THE APA}

The energy conservation relation is presented in [7], which was used to construct a unified framework to analyze the mean-square error (MSE), tracking, and transient characteristics. And this framework does not restrict the signal to specific models or to a Gaussian distribution. But the framework presented in [7] does not consider the nonstationarities of the signal model, which is our concern. So we should first extend the energy conservation relation in [7] to the condition of random and cyclic nonstationarities. is [2]

The update equation of the affine projection adaptive filter

$$
\mathbf{w}(k+1)=\mathbf{w}(k)+\mu \mathbf{S}(k)\left(\mathbf{S}^{H}(k) \mathbf{S}(k)\right)^{-1} \mathbf{e}^{*}(k)
$$

where $\mathbf{S}(k)=[\mathbf{s}(k), \mathbf{s}(k-1), \ldots, \mathbf{s}(k-M)]$ is the input data matrix, and $\mathbf{e}(k)=\mathbf{d}(k)-\mathbf{S}^{T}(k) \mathbf{w}^{*}(k)$ is the output error vector at the time of $k . M$ is the order of the affine projection adaptive filter. Obviously, if $M=0$, Eq. (4) is simplified as $\mathbf{w}(k+1)=\mathbf{w}(k)+\mathbf{s}(k)\left(\mathbf{s}^{H}(k) \mathbf{s}(k)\right)^{-1} \mathrm{e}^{*}(k)$, which is the update equation of the NLMS adaptive filter. So that, the affine projection filter is the extension of NLMS filter [12].

Let $\boldsymbol{\varepsilon}(k)$ denote the weight-error vector of the filter

$$
\boldsymbol{\varepsilon}(k)=\mathbf{w}_{o}(k) e^{j \omega k}-\mathbf{w}(k)
$$

where $\mathbf{w}(k)$ is the weight vector of the adaptive filter of order $L$ equal to the unknown channel $\mathbf{w}_{o}(k)$. Then, the steady-state MSE can be expressed as

$$
\begin{aligned}
\mathrm{MSE} & =\lim _{k \rightarrow \infty} E\left|d(k)-\mathbf{w}^{H}(k) \mathbf{s}(k)\right|^{2} \\
& =\lim _{k \rightarrow \infty} E\left|\boldsymbol{\varepsilon}^{H}(k) \mathbf{s}(k)+n(k)\right|^{2}=\zeta+\sigma_{n}^{2}
\end{aligned}
$$

where $\zeta=\lim _{k \rightarrow \infty} E\left|\boldsymbol{\varepsilon}^{H}(k) \mathbf{s}(k)\right|^{2}$ is the excess MSE (EMSE), and it can be seen that: when the variance of the noise $\sigma_{n}^{2}$ is constant, the evaluation of MSE is equal to the evaluation of EMSE. Substituting Eq. (5) into Eq. (4) yields the updated weight-error vector

$$
\boldsymbol{\varepsilon}(k+1)=\boldsymbol{\varepsilon}(k)-\mu \mathbf{S}(k)\left(\mathbf{S}^{H}(k) \mathbf{S}(k)\right)^{-1} \mathbf{e}^{*}(k)+\mathbf{c}(k) e^{j \omega k}
$$

where, $\mathbf{c}(k)=\mathbf{w}_{o}\left(e^{j \omega}-1\right)+\theta(k)\left(\alpha e^{j \omega}-1\right)+\mathbf{q}(k)$. If we define a priori error vector $\mathbf{e}_{a}(k)=\mathbf{S}^{T}(k) \varepsilon^{*}(k)$ and a posteriori error vector $\mathbf{e}_{p}(k)=$ $\mathbf{S}^{T}(k)\left(\boldsymbol{\varepsilon}(k+1)-\mathbf{c}(k) e^{j \omega k}\right)^{*}$, and substitute them into Eq. (7), we get

$$
\boldsymbol{\varepsilon}(k+1)=\boldsymbol{\varepsilon}(k)-\mathbf{S}(k)\left(\mathbf{S}^{H}(k) \mathbf{S}(k)\right)^{-1}\left(\mathbf{e}_{a}(k)-\mathbf{e}_{p}(k)\right)^{*}+\mathbf{c}(k) e^{j \omega k}
$$

By evaluating the energies of both sides of the Eq. (8), we can get the energy-conservation relation

$$
\begin{aligned}
& \left\|\boldsymbol{\varepsilon}(k+1)-\mathbf{c}(k) e^{j \omega k}\right\|^{2}+\mathbf{e}_{a}^{H}(k)\left(\mathbf{S}^{H}(k) \mathbf{S}(k)\right)^{-1} \mathbf{e}_{a}(k) \\
& =\|\boldsymbol{\varepsilon}(k)\|^{2}+\mathbf{e}_{p}^{H}(k)\left(\mathbf{S}^{H}(k) \mathbf{S}(k)\right)^{-1} \mathbf{e}_{p}(k)
\end{aligned}
$$

It can be seen from Eq. (9) that, the energies of the weighterror vectors at two successive iterations are related to the weighted energies of the a priori and a posteriori estimation error vectors. Eq. (9) is the extension to the APA case of the energy-conservation relation derived in [4] for NLMS algorithm, and the extension to the nonstationary case of APA derived in [7]. $E\|\boldsymbol{\varepsilon}(k+1)\|^{2}=E\|\boldsymbol{\varepsilon}(k)\|^{2}$ is satisfied when the adaptive filter get into steady-state, so the energy-conservation relation is suitable for the analysis of the steady-state performance of the adaptive filter. And it can be seen that no approximations are used to establish the energy relation of Eq. (9), so it is very universal.

\section{Tracking Performance of the APA to CFO}

In order to simplify the derivation, we consider the CFO in this section only, i.e. setting $\alpha=0 、 \mathbf{q}(k)=\mathbf{0}_{L \times 1}$. Then $\mathbf{c}(k)$ is a constant

$$
\mathbf{c}(k) \equiv \mathbf{c}=\mathbf{w}_{o}\left(e^{j \omega}-1\right)
$$

Substituting Eq. (10) and $E\|\boldsymbol{\varepsilon}(k+1)\|^{2}=E\|\boldsymbol{\varepsilon}(k)\|^{2}$ in steady state into Eq. (9), and taking expectations of both sides, the energy conservation relation of Eq. (9) becomes

$$
\begin{aligned}
& 2 \mu \operatorname{Re} E\left\{\mathbf{e}_{a}^{H}(k)\left(\mathbf{S}^{H}(k) \mathbf{S}(k)\right)^{-1} \mathbf{e}(k)\right\} \\
& =2 \operatorname{Re} E\left\{\boldsymbol{\varepsilon}^{H}(k+1) \mathbf{c} e^{j \omega k}\right\}+\mu^{2} E\left\{\mathbf{e}^{H}(k)\left(\mathbf{S}^{H}(k) \mathbf{S}(k)\right)^{-1} \mathbf{e}(k)\right\}-\|\mathbf{c}\|^{2}
\end{aligned}
$$

According to the definitions of $\mathbf{e}(k)$ and $\mathbf{e}_{a}(k)$, it is easy to get the following relationship

$$
\mathbf{e}(k)=\mathbf{e}_{a}(k)+\mathbf{n}(k)+\xi(k)
$$

where $\xi(k)=(\mathbf{A}-\mathbf{I}) \mathbf{S}^{T}(k) \mathbf{w}_{o}{ }^{*} e^{-j \omega k}$ and $\mathrm{A}=\operatorname{diag}\left(\left[1, e^{j \omega}, \ldots, e^{j \omega M}\right]\right)$. If setting $\omega=0, \quad \mathbf{A}=\mathbf{I}_{L \times L}, \quad \xi(k)=\mathbf{0}_{L \times 1}$ is satisfied, or if the order of the APA is zero, i.e. $M=0$, the APA is simplified as NLMS, then $\boldsymbol{\xi}(k)=\mathbf{0}_{L \times 1}$ is also satisfied. Substituting Eq. (12) into Eq. (11), and using the assumption that $\mathbf{n}(k)$ is independent of the other signal, then the Eq. (11) can be simplified

$$
\begin{aligned}
& \left(2 \mu-\mu^{2}\right) E\left\{\mathbf{e}_{a}^{H}(k)\left(\mathbf{S}^{H}(k) \mathbf{S}(k)\right)^{-1} \mathbf{e}_{a}(k)\right\} \\
= & 2 \operatorname{Re} E\left\{\boldsymbol{\varepsilon}^{H}(k+1)\right\} \mathbf{c}^{j \omega k}+\mu^{2} E\left\{\mathbf{n}^{H}(k)\left(\mathbf{S}^{H}(k) \mathbf{S}(k)\right)^{-1} \mathbf{n}(k)\right\}-\|\mathbf{c}\|^{2} \\
& -\left(2 \mu-\mu^{2}\right) \operatorname{Re} E\left\{\mathbf{e}_{a}^{H}(k)\left(\mathbf{S}^{H}(k) \mathbf{S}(k)\right)^{-1} \xi(k)\right\} \\
& +\mu^{2} E\left\{\xi^{H}(k)\left(\mathbf{S}^{H}(k) \mathbf{S}(k)\right)^{-1} \xi(k)\right\}
\end{aligned}
$$

If the filter length, i.e. $L$, is long enough, and the communication signal is constant modular modulation, such as FSK, PSK and so on, then $\mathbf{S}^{H}(k) \mathbf{S}(k) \approx L{\sigma_{s}}_{s}^{2} \mathbf{I}_{L \times L}$ is satisfied. Under this condition, Eq. (13) can be simplified 


$$
\begin{aligned}
& \left(2 \mu-\mu^{2}\right) E\left\|\mathbf{e}_{a}(k)\right\|^{2} \\
& =\mu^{2}(M+1) \sigma_{n}^{2}+2 L \sigma_{s}^{2} \operatorname{Re} E\left\{\boldsymbol{\varepsilon}^{H}(k+1)\right\} \mathbf{c} e^{j \omega k}-L \sigma_{s}^{2}\|\mathbf{c}\|^{2} \\
& -\left(2 \mu-\mu^{2}\right) \operatorname{Re} E\left\{\mathbf{e}_{a}^{H}(k) \xi(k)\right\}+\mu^{2} E\|\xi(k)\|^{2}
\end{aligned}
$$

According to the work of [7], we have

$$
\left(2 \mu-\mu^{2}\right) E\left\|\mathbf{e}_{a}(k)\right\|^{2}=\left(1-(1-\mu)^{2(M+1)}\right) E\left|e_{a}(k)\right|^{2}
$$

And the steady-state performance of the APA is characterized by $E\left|e_{a}(k)\right|^{2}$. So, we have to solve $E\left|e_{a}(k)\right|^{2}$ according to Eq. (14). It can be seen from Eq. (14) that, in order to solve $E\left|e_{a}(k)\right|^{2}$, we have to solve $E\{\boldsymbol{\varepsilon}(k)\}, E\left\{\mathbf{e}_{a}{ }^{H}(k) \xi(k)\right\}$ and $E\|\xi(k)\|^{2}$ first. The process of solving them is tedious, so we give the results here only:

$$
\begin{gathered}
E\{\boldsymbol{\varepsilon}(k)\}=\left[\mathbf{I}-\mu \overline{\mathbf{R}}_{s}-e^{j \omega} \mathbf{I}\right]^{-1}\left[\frac{\mu}{L}\left(\frac{1-e^{-j \omega(M+1)}}{1-e^{-j \omega}}-(M+1)\right) \mathbf{w}_{o}-\mathbf{c}\right] e^{j \omega k} \\
E\|\xi(k)\|^{2}=2\left\|\mathbf{w}_{o}\right\|^{2}\left[M+1-\operatorname{Re} \frac{1-e^{-j \omega(M+1)}}{1-e^{-j \omega}}\right] \\
E\left\{\mathbf{e}_{a}^{H}(k) \xi(k)\right\}=\boldsymbol{\varepsilon}^{T}\left(\frac{1-e^{-j \omega(M+1)}}{1-e^{-j \omega}}-(M+1)\right) \sigma_{s}^{2} \mathbf{w}_{o}^{*}
\end{gathered}
$$

Substituting Eq. (15)-(18) into Eq. (14), we can get the expression of $E\left|e_{a}(k)\right|^{2}$ easily. Here, we are interested in two special cases:

(1) If setting $\omega=0$, which means there is no CFO in the transmitter and receiver carrier generators, then $\mathbf{c}=\mathbf{0}_{L \times 1}$ is satisfied. And we have the following limitation

$$
\lim _{\omega \rightarrow 0} \frac{1-e^{j \omega(M+1)}}{1-e^{j \omega}}=M+1
$$

Using (19), we can get Eq. (16)-(18) equal zeros. And substituting these results into Eq. (14), we can get

$$
E\left|e_{a}(k)\right|^{2}=\frac{\mu}{2-\mu} \sigma_{n}^{2}
$$

Eq. (20) is the same as the Eq. (22) in [7], which is the steady-state performance of the APA with no nonstationary.

(2) If setting $M=0$, the APA is simplified as the NLMS algorithm. And $E\|\xi(k)\|^{2}, E\left\{\mathbf{e}_{a}^{H}(k) \xi(k)\right\}$ is equal to zeros. Substituting these conditions into Eq. (14), we can get

$$
E\left|e_{a}(k)\right|^{2}=\frac{\mu}{2-\mu} \sigma_{n}^{2}+\frac{L \sigma_{s}^{2}}{2 \mu-\mu^{2}}\left[2 \operatorname{Re}\left(\boldsymbol{\varepsilon}^{H} \mathbf{c} e^{-j \omega}\right)-\|\mathbf{c}\|^{2}\right]
$$

Eq. (21) is the same as the result in Table III in [4], which is the steady-state performance of the NLMS algorithm with cyclic nonstationarities.

\section{Simulation Results}

In order to evaluate the validation of the theoretical results derived in section III, we compare them with 4 group numerical results in this section. The parameters are set in
Table 1. Apart from these parameters, the communication signals are Binary PSK (BPSK) modulated signal with power $\sigma_{s}^{2}=1$ watt. Because the tracking performance of the adaptive is belong to the steady-state performance, the weights of the affine projection adaptive filter are initialized as the actual channel parameters. Monte Carlo experiments are carrier out with each result averaged over 100 independent runs, and the number of the transmitted signals is 1000 .

TABLE I. PARAmeters Setting OF The Simulations

\begin{tabular}{|c|c|c|c|c|}
\hline Simulation & $\mathbf{1}$ & $\mathbf{2}$ & $\mathbf{3}$ & $\mathbf{4}$ \\
\hline Parameter & & & & \\
\hline \multirow{2}{*}{$(\mathbf{r a d} / \mathbf{s y m})$} & $0.001,0.005$, & 0.001, & 0.001, & 0.01, \\
& $0.01,0.0005$ & $0.005,0.01$ & 0.01 & 0.03 \\
\hline $\boldsymbol{L}$ & 51 & 51 & 10 & 10 \\
\hline $\mathbf{S N R}(\mathbf{d B})$ & 30 & 30 & 30 & 10 \\
\hline $\boldsymbol{M}$ & 2 & 4 & 2,4 & 2,4 \\
\hline
\end{tabular}

The theoretical results of $E\left|e_{a}(k)\right|^{2}$ in Eq. (15) and Monte Carlo simulation curves are plotted in Fig. 2. It is seen that when the step size $\mu$ is small, the theoretical results are very close to the simulation curves. The difference between the theoretical results and the simulation curves is increased along with the increase of the step size. This is due to the approximation used in the deriving process.

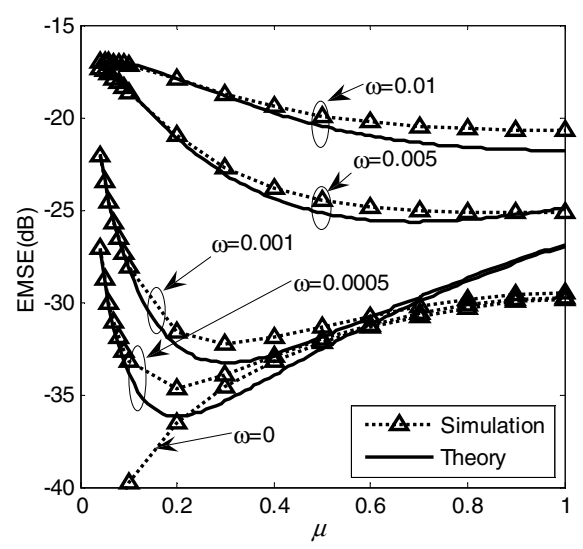

Figure2. Theoretical results vs. simulation curves of APA(2).

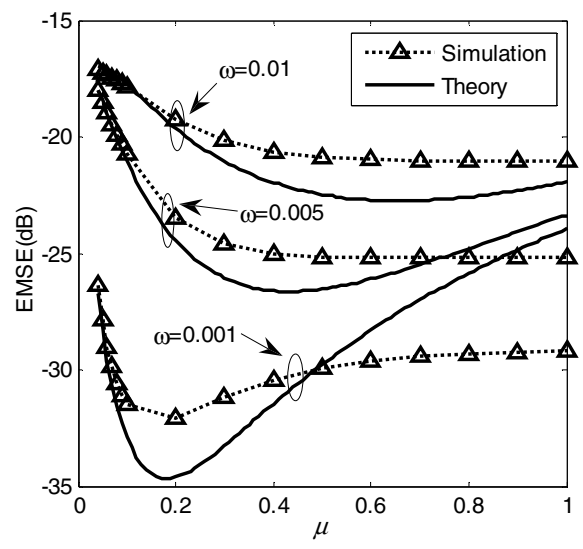

Figure3. Theoretical results vs. simulation curves of APA(4).

It has been concluded that, when there is no nonstationarities, the extended mean-square error originates from the gradient noise. And EMSE increases according to the 
step-size $\mu$ [2]. But it can be seen from Fig. 2 that this conclusion is changed when there is CFO. When $\mu$ is small, the adaptive filter cannot track the CFO effectively, and the EMSE is due to the difference between the filter and the time-variation system mainly. As the increase of the step-size $\mu$, the tracking capacity of the adaptive filter improves. And the EMSE decrease accordingly. But as the step-size increases continue, the gradient noise becomes the major factor of the EMSE, and the EMSE increase again. So there exists the best step-size, with which the adaptive filter achieves the smallest EMSE, for example, $\omega=0.005,0.001$ and 0.0005 . But when the CFO is so large that the adaptive filter cannot track the system effectively, the EMSE will decrease as the increase of $\mu$, for example, $\omega=0.01$.

It can be drawn the same conclusion from Fig. 3 as get from Fig. 2. And compared Fig. 3 with Fig. 2, it can be seen that the difference between theoretical results and simulation curves increases according the order of the APA.

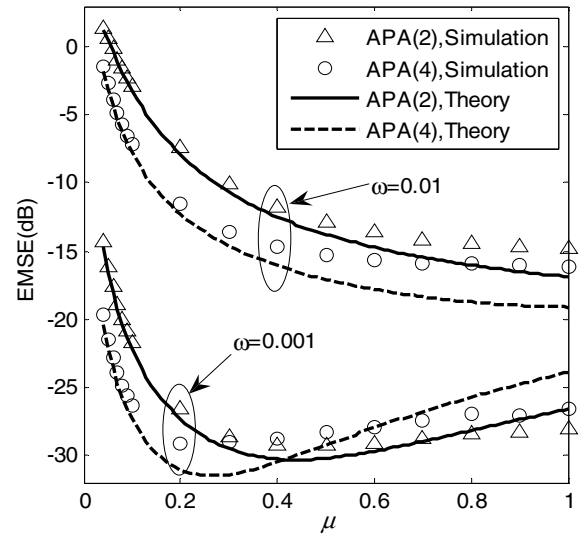

Figure4. Tracking performance of the APA with different order.

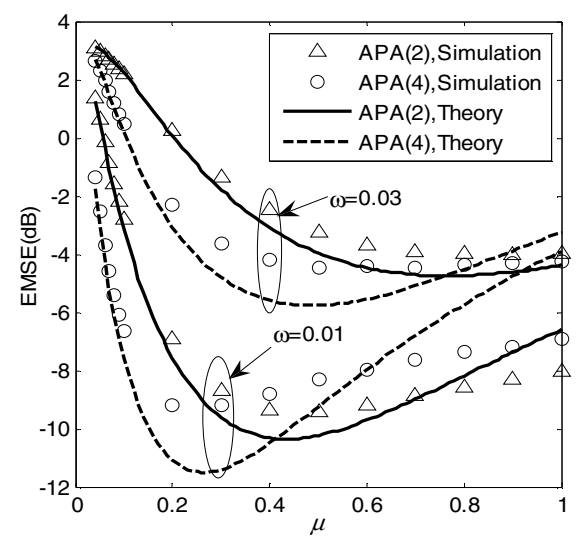

Figure5. Tracking performance of the APA in low SNR.

Figure 4 shows the tracking performance of the APA with different order. It is seen from $\omega=0.01$ that the APA with order $M=4$ has lower EMSE than the APA with order $M=2$ all the time. So it can be concluded that the tracking ability of the APA enhanced according to the increase of the order. It is seen from $\omega=0.001$ that the APA with order $M=4$ has lower EMSE than the APA with order $M=2$ when the step-size is small. While EMSE of the APA with order $M=4$ go to higher than the
APA with order $M=2$. This is because the gradient noise increases according to the order of the algorithm. So that, when there is CFO, the order of the algorithm and the step-size should be optimized at the same time to minimize the EMSE.

Figure 5 shows the tracking performance of the APA in low SNR environment and high CFO. It is seen that the theoretical results are close to the simulations curves even in the condition of low SNR and high CRO.

\section{CONCLUTIONS}

In this paper, we analyzed the tracking performance of the affine projection filter in the presence of carrier frequency offset. The energy-conservation relation of the affine projection algorithm presented in [7] is extended to adapt to random and cyclic nonstationarities environments. Then the theoretical expression of steady-state mean-square error is derived based on the energy-conservation relation. Several simulations show that the theory results approach the practical situation very well. And it can be concluded that for moderate carrier frequency offset, there is the best step-size to minimize the steady-state mean-square error. Future work will address how to adjust the parameters of the affine projection filter to the time-varying environments adaptively, and what degree the carrier frequency offsets affects the bit-error-rate when there is adaptive filter in the communication system.

\section{REFERENCES}

[1] A. F. Molisch. Wideband wireless digital communications [M]. Englewood Cliffs, NJ: Prentice-Hall, 2001.

[2] S. Haykin. Adaptive filter theory [M], 4th ed. Englewood Cliffs, NJ: Prentice-Hall, 2002.

[3] M. Hajivandi, W. A. Gardner. Measures of tracking performance for the LMS algorithm [M]. IEEE Transactions on Acoustic, Speech and Signal Processing, 1990, 38(12): 1953-1958.

[4] N. R. Yousef, A. H. Sayed. Ability of adaptive filters to track carrier offsets and random channel nonstationarities [J]. IEEE Transactions on Signal Processing. 2002, 50(7): 1533-1544.

[5] M. Moinuddian, A. Zerguine. Tracking analysis of the NLMS algorithm in the presence of both random and cyclic nonstationarities [J]. IEEE Signal Processing Letters, 2003, 10(9): 256-258.

[6] J. M. Gorriz, J. Ramirez, S. C. Alvarez, etc. A Novel LMS algorithm applied to adaptive noise cancellation [J]. IEEE Signal Processing Letters, 2009, 16(1): 34-37.

[7] H. C. Shin, A. H. Sayed. Mean-square performance of a family of affine projection algorithm [J]. IEEE Transactions on Signal Processing. 2004, 52(1): 90-101.

[8] H. C. Shin, W. J. Song, A. H. Sayed. Mean-square performance of datareusing adaptive algorithms [J]. IEEE Signal Processing Letters, 2005, 12(12): 851-854.

[9] H. Rey, L. Rey Vega, S. Tressens. Variable explicit regularization in affine projection algorithm: robustness issues and optimal choice [J]. IEEE Transactions on Signal Processing, 2007, 55(5): 2096-3109.

[10] Y. Zhu, Y. Zhu, F. Yao, etc. A performance study of affine projection adaptive equalizers in the presence of strong narrowband interference [C]. Kunming :CMC2009: II-386-390.

[11] Y. Zhu, F. Yao, Y. Zhu, etc. A novel affine projection algorithm with dynamic selection of input vectors [J]. Journal of System Simulation, 2010, 22(4): 2215-2218.

[12] Y. Zhu, F. Yao, L. Chen, etc. The uniform framework of variable stepsize adaptive filter algorithms and its extension to vector region [J]. Signal Processing, 2009, 25(10): 1542-1549. 